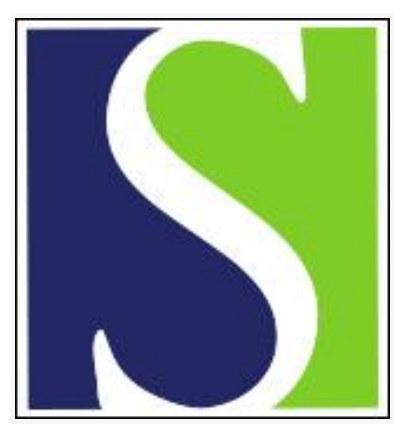

Scand J Work Environ Health 2006;32(5):368-373

https://doi.org/10.5271/sjweh.1032

Issue date: 31 Oct 2006

Cancer incidence among physicians occupationally exposed to ionizing radiation in Finland

by Jartti P, Pukkala E, Uitti J, Auvinen A

Affiliation: Tampere School of Public Health, 33014 University of Tampere, Tampere, Finland. pirkko.jartti@uta.fi

Key terms: cancer incidence; Finland; ionizing radiation; neoplasm; occupational exposure; physician; radiation effect; radiology; X-ray

This article in PubMed: www.ncbi.nlm.nih.gov/pubmed/17091204

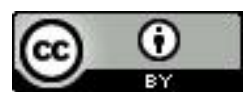




\title{
Cancer incidence among physicians occupationally exposed to ionizing radiation in Finland
}

\author{
by Pirkko Jartti, MD, 1,2 Eero Pukkala, PhD, ${ }^{3}$ Jukka Uitti, MD, ${ }^{2,4}$ Anssi Auvinen, MD ${ }^{1,5}$
}

\begin{abstract}
Jartti P, Pukkala E, Uitti J, Auvinen A. Cancer incidence among physicians occupationally exposed to ionizing radiation in Finland. Scand J Work Environ Health 2006;32(5):368-373.
\end{abstract}

\begin{abstract}
Objectives Occupational radiation exposure was estimated, and the cancer incidence among physicians working with radiation was compared to that of unexposed physicians.

Methods A cohort of 1312 physicians was identified from the Finnish occupational radiation exposure registry. Radiation exposure data were obtained from 1970 to 2001 on the basis of individual dosimeters. Nevermonitored Finnish physicians $(\mathrm{N}=15$ 821) were used as a reference group, identified from census data of Statistics Finland. Incident cancer cases were identified by record linkage with the Finnish Cancer Registry.

Results The cumulative radiation dose exceeded the recording level (0.3-3.0 mSv during a 3-month period for 1029 radiation-exposed physicians (59.8\%). Six percent of the radiologists had received a cumulative dose of 50 $\mathrm{mSv}$ or more. Altogether there were 41 cancers observed among the radiation-exposed physicians and 998 cases found in the never-monitored group. Standardized incidence ratios (SIR) for all cancers were comparable with those of the general population among physicians monitored for radiation [SIR 1.0, 95\% confidence interval (95\% CI) 0.7-1.4] and other physicians (SIR 1.0, 95\% CI 1.0-1.1). For specific cancer sites, a slightly elevated risk of female breast cancer was found among monitored physicians when compared with other physicians (rate ratio 1.7, 95\% CI 1.0-3.1). No obvious dose-response relationship was found for the overall cancer incidence.

Conclusions According to the results from a nationwide cohort, occupational exposure to medical radiation is not a strong risk factor for cancer among physicians. Possible excess risk could not be reliably demonstrated even after the follow-up of a nationwide cohort for up to 30 years.
\end{abstract}

Key terms neoplasms; occupational exposure; radiation effects; radiology; X-rays.

Cancer risk has been studied in several occupational groups exposed to ionizing radiation, including radiological workers and nuclear industry and airline personnel. An international study evaluated cancer mortality among 95000 nuclear power plant workers in the United States, the United Kingdom, and Canada (1). Mortality due to leukemia was related to radiation dose, and the result was consistent with the studies of atomic bomb survivors. Another large cohort study investigated the relation between cancer incidence and occupational exposure to ionizing radiation among 190000 Canadian radiation workers (2). The incidence of thyroid cancer and melanoma was increased in the occupationally exposed cohort. A study of 10000 Nordic airline pilots did not indicate a markedly increased cancer risk attributable to cosmic radiation (3). However, the relative risk of skin cancers increased with the estimated radiation dose.

Physicians working with radiological imaging were one of the first groups exposed to radiation. Before the era of the first radiological protection recommendations in the 1920s, radiologists were exposed to high cumulative doses of ionizing radiation, up to several Gray. In 50 years of follow-up of British radiologists, mortality due to cancer was $75 \%$ higher among early radiologists than among medical practitioners in general (4). An excess risk was found for leukemia [standardized mortality ratio (SMR) 1.88, 95\% confidence interval

1 Tampere School of Public Health, University of Tampere, Tampere, Finland.

2 Tampere Regional Institute of Occupational Health, Tampere, Finland.

3 Finnish Cancer Registry, Helsinki, Finland.

4 Clinic of Occupational Medicine, Tampere University Hospital, Tampere, Finland.

5 STUK-Radiation and Nuclear Safety Authority, Helsinki, Finland.

Reprint requests to: Professor Anssi Auvinen, Tampere School of Public Health, 33014 University of Tampere, Tampere, Finland. [E-mail: anssi.auvinen@uta.fi] 
(95\% CI) 0.81-3.70] and skin cancer (SMR 1.09), but lung and pancreatic cancer deaths were also increased. No evidence of increased cancer mortality was observed among radiologists registered for the first time after 1954, probably due to improved radiation protection. In an American cohort study, cancer mortality rates were higher for radiologists joining a radiological society prior to 1950 than those of other specialists (5). Cancer mortality has been studied also among 27000 medical $\mathrm{X}$-ray workers in China (6). Similarly, an increased risk for leukemia and solid cancer was found in the earlier cohort (before 1970), but not for workers first employed after 1970.

In a nationwide cohort of almost 150000 radiology technologists in the United States, overall mortality, as well as cancer mortality, was low. However, mortality from breast cancer and leukemia (excluding chronic lymphocytic leukemia) increased with duration of employment (7). Among 90000 radiology technologists in the United States who were followed during 1983-1998, the elevated risk for breast cancer, leukemia, and basalcell skin carcinoma may have been related to occupational radiation exposure (8-10).

None of the previous studies on occupational exposure to the medical use of radiation has been able to use individual radiation dose (dosimeter readings). In Finland, occupational radiation exposure is monitored by STUK (Radiation and Nuclear Safety Authority) and a dose registry established covering dose estimates for all medical radiation workers in Finland for more than 30 years. We undertook this study to assess the relationship between cumulative radiation exposure and cancer incidence among Finnish physicians involved in X-ray diagnostics and radiotherapy (radiologists, cardiologists, intervention radiologists, oncologists, and physicians of other specialties), using never-monitored Finnish physicians as a reference group.

\section{Study population and methods}

\section{Study population}

We identified 1312 physicians with valid personal identification numbers from the Finnish occupational radiation exposure registry of STUK (Radiation and Nuclear Safety Authority). Their radiation exposure has been monitored using personal film or thermoluminescent dosimeters with readings recorded every 3 months for the years 1970-2001. As zero doses were not recorded prior to 1990 , the start or length of the monitoring period was not available. Of the monitored physicians, 70\% (920) were men and 30\% (392) were women. The largest subgroup comprised radiologists (51\%), followed by surgeons $(30 \%)$, cardiologists $(8 \%)$, intervention radiologists (2\%), oncologists (1\%), and physicians of other specialties $(8 \%)$.

A reference cohort was formed comprising a $90 \%$ random sample of nonmonitored physicians, identified from the six population censuses carried out between 1970 and 1995, available from Statistics Finland. The reference cohort consisted of 15281 physicians (7112 women and 8169 men), for whom date of birth, gender, and occupation were available (separately for 1970, 1975, 1980, 1985, 1990, and 1995). Physicians included in the exposed group were excluded from the reference cohort.

\section{Follow-up}

For the exposed group, follow-up started at the first known date of dose monitoring, typically the first date with a dosimeter reading exceeding the recording threshold. For the reference group, the start of followup was defined as the date of the census in which they were first identified as physicians. The dates of death and emigration were obtained from Statistics Finland. For both cohorts, the follow-up ended at the date of death, emigration, or cancer diagnosis or the common closing date (December 2001).

Cancer diagnoses among the exposed and reference cohorts were obtained by linkage to the Finnish Cancer Registry, which is a nationwide population-based cancer registry with practically complete coverage (11). We studied the incidence of all cancers, and the primary end point was the incidence of the cancers most strongly associated with radiation in previous studies (squamouscell skin cancer, basal-cell carcinoma, thyroid carcinoma, breast cancer, and leukemia). Other primary sites were also analyzed, but they were regarded as secondary end points and interpreted as exploratory analyses. Information was obtained on the date of cancer diagnosis, primary site, histological type, and malignancy.

\section{Statistical methods}

The expected numbers of cancer cases were calculated on the basis of national age- and gender-specific cancer incidence rates during the study period. Standardized incidence ratios (SIR) were calculated as the ratio of the observed to the expected numbers of cases. The confidence interval $(95 \% \mathrm{CI})$ for the standardized incidence ratio was calculated on the assumption that the observed numbers of cases follow a Poisson distribution. The exact method was used when there were fewer than five observed cases. The incidence of selected cancer types in the exposed group, as compared with the never-monitored physicians, was assessed using Poisson regression methods (12). 


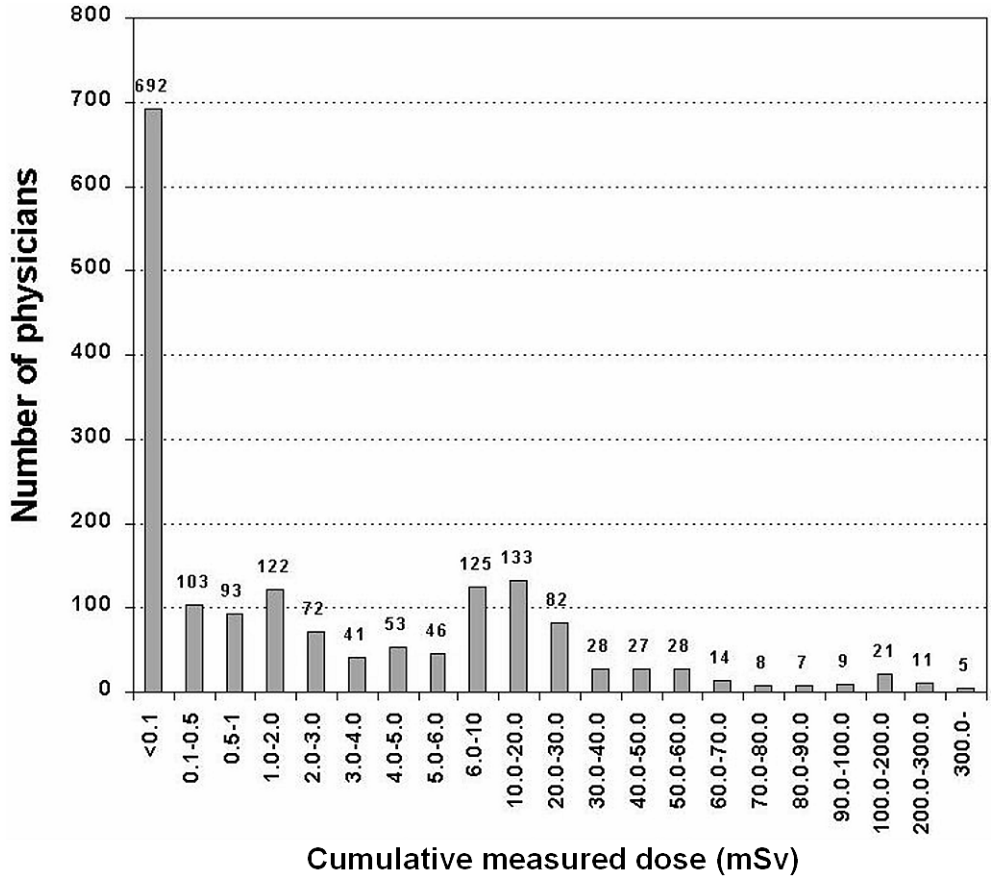

Figure 1. Distribution of cumulative measured radiation doses (mSv) between 1970 and 2001 among Finnish physicians monitored for radiation exposure.
Table 1. Number of persons and person-years by gender among the Finnish physicians monitored for radiation exposure and a reference group of nonmonitored physicians.

\begin{tabular}{lcr}
\hline Group & $\begin{array}{c}\text { Number of } \\
\text { persons }\end{array}$ & $\begin{array}{c}\text { Person- } \\
\text { years }\end{array}$ \\
\hline $\begin{array}{l}\text { Physicians monitored for } \\
\text { radiation (N=1312) }\end{array}$ & \\
$\quad$ Men & 920 & 10329 \\
$\quad$ Women & 392 & 4095 \\
Physicians not monitored for & & \\
radiation exposure (N=15 281) & & \\
$\quad$ Men & 8169 & 162960 \\
$\quad$ Women & 7112 & 117057 \\
\hline
\end{tabular}

We did not have information on time of graduation or licensing, or on the first date of monitoring (or when the physician started working). However, we carried out additional analyses by year of birth, assuming that most physicians monitored prior to 1970 were born in 1950 or earlier.

The study protocol was approved by the ethical review board of the Pirkanmaa Hospital District, and permission to use the registry data was obtained from STAKES-Research and Development Centre for Welfare and Health.

\section{Results}

The cumulative radiation doses were below the recording level (3.0 mSv for the 3-month period in 1970-1974,
$1.5 \mathrm{mSv}$ for $1975-1979,0.5 \mathrm{mSv}$ for $1980-1988,0.3$ $\mathrm{mSv}$ per 3 months from 1989 on) for $40.2 \%$ of the physicians in the study cohort (figure 1) The mean dose was $12.7 \mathrm{mSv}$, with a median of $1 \mathrm{mSv}$. Six percent of the monitored physicians received a cumulative dose of 50 $\mathrm{mSv}$ or more. Sixteen physicians had cumulative doses exceeding $200 \mathrm{mSv}$, the highest cumulative dose being $942 \mathrm{mSv}$. The highest mean doses were recorded for intervention radiologists $(61 \mathrm{mSv})$, followed by cardiologists $(28 \mathrm{mSv})$ and radiologists $(12 \mathrm{mSv})$.

The mean length of follow-up for the 1312 physicians working with radiation was 11 years, while that for the 15281 reference physicians $(8169$ men and 7112 women) was 18 years (table 1). Among the physicians monitored for radiation exposure, 22 cancers were diagnosed among the men and 19 were recorded for the women (table 2). Correspondingly, there were 998 cancers in the reference group (590 among the men and 408 among the women).

The overall cancer incidence was higher among the female physicians in both the exposed and the reference groups than in the age-matched Finnish female population in general (SIR 1.71, 95\% 0.91-2.91, and 1.24, 95\% CI 1.12-1.35, respectively). For the male physicians, a nonsignificantly lower cancer incidence was found than for the entire Finnish population (SIR 0.74, 95\% CI $0.46-1.11$, for monitored and $0.93,95 \%$ CI $0.86-1.00$, for nonmonitored). Of the specific cancer sites, the physicians monitored for radiation exposure had a significantly increased incidence of breast cancer and melanoma. No cases of lung cancer or nonmelanoma skin cancer occurred in this group. Similar to the 
Table 2. Standardized incidence ratio (SIR) and incidence rate ratio for cancer among Finnish physicians monitored for radiation exposure and a reference group of nonmonitored physicians. ( $S I R$ = standardized incidence ratio relative to the entire Finnish population, rate ratio $=$ SIR of exposed physicians relative to the SIR of never-monitored physicians)

\begin{tabular}{|c|c|c|c|c|c|c|c|c|c|c|}
\hline \multirow[t]{2}{*}{ Cancer site } & \multicolumn{4}{|c|}{$\begin{array}{l}\text { Cases among physicians } \\
\text { monitored for radiation }\end{array}$} & \multicolumn{4}{|c|}{$\begin{array}{l}\text { Cases among physicians } \\
\text { not monitored for radiation }\end{array}$} & \multirow[t]{2}{*}{ Rate ratio } & \multirow[t]{2}{*}{$95 \% \mathrm{Cl}$} \\
\hline & Observed & Expected & SIR & $95 \% \mathrm{Cl}$ & Observed & Expected & SIR & $95 \% \mathrm{Cl}$ & & \\
\hline Thyroid & 2 & 0.9 & 2.4 & $0.3-8.5$ & 29 & 18 & 1.6 & $1.1-2.3$ & 1.5 & $0.4-6.3$ \\
\hline Leukemia & 2 & 0.9 & 2.4 & $0.3-8.2$ & 11 & 21 & 0.5 & $0.3-0.9$ & 4.4 & $0.5-20$ \\
\hline Breast & 12 & 4.7 & 2.3 & $1.3-4.4$ & 181 & 124 & 1.5 & $1.3-1.7$ & 1.7 & $1.0-3.1$ \\
\hline Skin, oat cell & - & 0.8 & 0 & $0.0-4.6$ & 30 & 23 & 1.3 & $0.9-1.9$ & 0 & $0-3.8$ \\
\hline Skin, basal cell & 7 & 8.0 & 0.9 & $0.4-1.8$ & 311 & 175 & 1.8 & $1.6-2.0$ & 0.5 & $0.2-1.0$ \\
\hline All cancers & 41 & 41 & 1.0 & $0.7-1.4$ & 998 & 964 & 1.0 & $1.0-1.1$ & 1.0 & $0.7-1.3$ \\
\hline
\end{tabular}

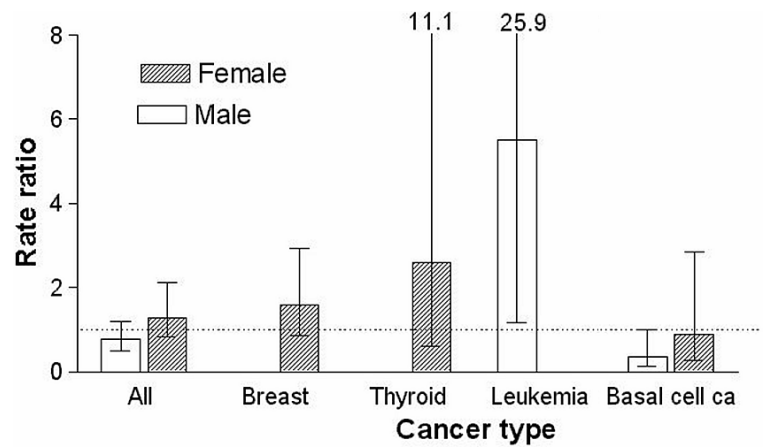

Figure 2. Rate ratio by cancer type with the $95 \%$ confidence interval for male and female Finnish physicians monitored for radiation exposure relative to other physicians. $(\mathrm{ca}=$ cancer $)$

situation in the exposed group, excess risks of breast cancer and melanoma were also found in the reference cohort, as well as an increased incidence of thyroid cancer.

When the physicians monitored for radiation were compared with the reference physicians, no overall excess of cancer risk was found. However, the incidence of leukemia was higher among the monitored than among the nonmonitored men [rate ratio (RR) 5.5, 95\% CI 1.2-25.9], while no such increase was found for the women (no cases among the monitored physicians) (figure 2). In addition, the risk of melanoma was increased for the monitored women (RR 4.2, 95\% CI 1.3-14.2), but not for the monitored men (RR 1.6, 95\% CI 0.55.4).

Cancer incidence did not differ substantially among the monitored physicians born in 1950 or earlier (presumed to have started working prior to 1970) from those born later (for whom the entire exposure history was likely to be available) (SIR 0.95 versus 1.20 , RR 0.95 , 95\% CI 0.47-1.88). The monitored physicians born before 1951 had a significant excess of breast cancer (SIR $3.1,95 \%$ CI $1.55-5.94$, on the basis of 10 cases). The excess of melanoma was confined to physicians working

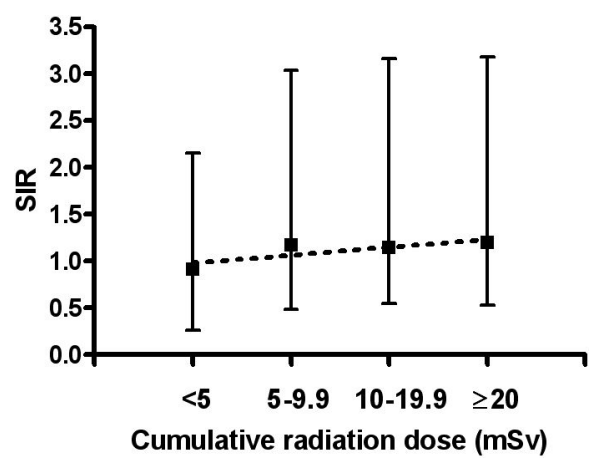

Figure 3. Standardized incidence ratios (SIR) for cancer and their $95 \%$ confidence intervals (error bars) according to the cumulative measured radiation dose among Finnish physicians. The slope of the regression indicates a nonsignificant increase in the relative risk of cancer per $10 \mathrm{mSv}$ of radiation exposure.

with radiation who had started their career after 1970 (SIR 7.1, 95\% CI 1.93-18.12, on the basis of four cases).

Cancer risk was evaluated in relation to the radiation dose. The standardized incidence ratios for the physicians with cumulative radiation doses of $<5,5-9.9$, $10-19.9$, and $>20 \mathrm{mSv}$ were $0.91,1.17,1.15$, and 1.20 , respectively (figure 3 ). A linear regression analysis indicated a $9 \%$ excess relative risk per $10 \mathrm{mSv}$ of radiation (95\% CI -20\%-40\%), which does not differ statistically significantly from zero.

\section{Discussion}

We conducted a cohort study of cancer incidence among Finnish physicians monitored for radiation exposure and compared their cancer incidence with that of other physicians. We found only minor differences in the cancer incidence between the physicians potentially exposed to radiation and other physicians. This finding suggests that occupational exposure to low doses of radiation 
among physicians does not cause a major increase in cancer burden. On the basis of the size of the study cohort, we estimate that it was possible to exclude a relative excess risk of $30 \%$ or higher. Due to the relatively small numbers of physicians with radiation doses exceeding $10 \mathrm{mSv}$, it was not possible to estimate the dose-response meaningfully.

Globally, the average annual radiation doses among the monitored physicians decreased from 0.8 to $0.4 \mathrm{mSv}$ between 1975 and 1994 (13). In Finland, no obvious trends for the mean annual doses of physicians working with radiation have been recorded, and this situation mainly reflects the fact that the annual doses had been very low already in the 1970s (13). The radiation dose from occupational exposure is a relatively small addition to radiation exposure received from other sources, including environmental sources and medical uses (the mean annual dose to the Finnish population being 4 $\mathrm{mSv}$, with $2 \mathrm{mSv}$ from indoor radon and $0.5 \mathrm{mSv}$ from medical uses of radiation). In our study, the mean recorded cumulative dose was $12 \mathrm{mSv}$, and only $6 \%$ of the physicians had received a cumulative recorded dose of $50 \mathrm{mSv}$ or higher. No doses reaching the recording level (which decreased from 1.5 to $0.3 \mathrm{mSv}$ per 3-month period) were recorded for $40 \%$ of the physicians. It should also be noted that the dosimeter is generally worn above any radioprotective shield such as a lead apron. Therefore, the doses are likely to be overestimated by at least one order of magnitude.

Lack of information on the starting date of monitoring and below threshold doses makes it difficult to estimate the extent of missed doses due to changes in the recording level. However, previous studies have shown that, in most circumstances, missing doses that are below the threshold of detection do not strongly bias the results $(14,15)$. Yet more important than the changes in the recording level was the lack of information on exposures before 1970. Even though most of the monitored physicians had their first recorded dose well after 1970 , the cancer incidence of the late entry group with a more complete exposure history was comparable to that of the older cohort with a longer working history and probably higher cumulative doses. More than three quarters of the 41 cancer cases occurred in the group that started their work before 1970, the finding reflecting the young age and low cancer risk in the late entry cohort. This situation limits the usefulness of the recorded doses. However, further follow-up will allow analyses confined to the cohort for which a more complete exposure history is available.

Cancer risk among physicians working with radiation is not only affected by their radiation exposure, but also very strongly by lifestyle factors such as tobacco, diet, and reproductive history. The incidence of several cancers (lung cancer in particular) among physicians is below that of the rest of the population (16-18). The difference partly remains even in comparison with other occupations in the highest social class. To minimize confounding due to factors other than occupational radiation exposure, we used a reference group comprised of physicians not exposed to occupational radiation. This approach has been utilized also in some previous studies $(4,5)$.

The minimum length of the latency period (ie, the time from radiation exposure to the manifestation of its effects) is probably highly variable, ranging from a few years for leukemia to up to a decade for most solid cancers. In our study, the mean length of follow-up in the exposed group was 11 years. It is therefore possible that some of the risk might not have been detected due to a relatively short follow-up. However, cancer incidence among the physicians likely to have started their career prior to 1970 did not substantially differ from that of the physicians first exposed after 1970.

Our study differed from most previously published reports in several aspects. First, we studied cancer incidence and not cancer mortality. When reliable registries are available, cancer incidence is a better indicator of cancer risk, since a large and increasing proportion of cancers are not lethal. This situation is particularly important for cancer types with little excess mortality, such as thyroid cancer and nonmelanoma skin cancer. In this study, we paid particular attention to nonmelanoma skin cancers, which often occur in the extremities. In intervention radiology, the radiation beam may occasionally reach the hands of the radiologists, leading potentially to high local radiation doses. Few studies have systematically assessed the typical hand doses for physicians working with radiation $(19,20)$. Finger doses were not recorded by the Finnish dose register. As detailed information on both squamous cell carcinoma and basal cell carcinoma is available from the Finnish Cancer Registry, we were able to conclude that no malignant nonmelanoma skin cancers were found among monitored physicians. The incidence of basal-cell cancer was actually below that of the other physicians, comparable with the age-matched Finnish population in general.

The lack of demonstrable cancer risk in a comprehensive nationwide cohort suggests that any excess cancer risk is likely to be small. This low risk is probably due to improved radiation protection resulting in low cumulative doses for the cohort. This finding is consistent with the findings of previous studies, showing no increase in cancer mortality in groups occupationally exposed in conjunction with medical uses of radiation after 1970 (4-6). Yet, 6\% of the physicians had accrued high radiation doses even during the past 10 years, the situation suggesting that continued surveillance and further improvements in radiation protection are still warranted. 


\section{Acknowledgments}

The study was financially supported by a grant from the Finnish Work Environment Fund (grant 102094).

We wish to thank Eija Vartiainen, MSc, from the STUK-Radiation and Nuclear Safety Authority, for providing the dose data, as well as Jari Nieminen and Jari Tarkoma, from Statistics Finland, for establishing the reference cohort.

\section{References}

1. Cardis E, Gilbert ES, Carpenter L, Howe G, Kato I, Armstrong BK, et al. Effects of low doses and low dose rates of external ionizing radiation: cancer mortality among nuclear industry workers in three countries. Radiat Res. 1995;142:117-32.

2. Sont WN, Zielinski JM, Ashmore JP, Jiang H, Krewski D, Fair ME, et al. First analysis of cancer incidence and occupational radiation exposure based on the national dose registry of Canada. Am J Epidemiol. 2001;4:309-18.

3. Pukkala E, Aspholm R, Auvinen A, Eliasch H, Gundestrup M, Haldorsen $\mathrm{T}$, et al. Incidence of cancer among Nordic airline pilots over five decades: occupational cohort study. Br Med J. 2002;325:567.

4. Berrington A, Darby S, Weiss H, Doll R. 100 years of observation on British Radiologists: mortality from cancer and other causes 1897-1997. Br J Radiol. 2001;74:507-19.

5. Matanoski GM, Seltser R. Sartwell PE, Diamond EL, Elliott EA. The current mortality rates of radiologists and other physician specialists. Deaths from all causes and from cancer. Am J Epidemiol. 1975;101:188-98.

6. Wang JX, Zhang LA, Li BX, Zhao YC, Wang ZQ, Zhang JY, et al. Cancer incidence and risk estimation among medical Xray workers in China 1950-1995. Health Phys. 2002;82:45566.

7. Mohan AK, Hauptmann M, Freedman MD, Ron E, Matanoski GM, Lubin JH, et al. Cancer and other causes of mortality among radiologic technologists in the United States. Int J Cancer. 2003;103:259-67.
8. Sigurdson AJ, Doody MM, Rao RS, Freedman DM, Alexander $\mathrm{BH}$, Hauptmann M, et al. Cancer incidence in the US radiologic technologists health study, 1983-1998. Cancer 2003;97:3080-9.

9. Yoshinaga S, Hauptmann M, Sigurdson AJ, Doody MM, Freedman DM, Alexander BH, et al. Nonmelanoma skin cancer in relation to ionizing radiation exposure among U.S. radiologic technologists. Int J Cancer 2005;115:828-34.

10. Linet MS, Freedman DM, Mohan AK, Doody MM, Ron E, Mabuchi K, et al. Incidence of haematopoietic malignancies in US radiologic technologists. Occup Environ Med. 2005;62:861-7.

11. Teppo L, Pukkala E, Lehtonen M. Data quality and quality control in a population-based cancer registry. Acta Oncol. 1994:33:365-9.

12. Breslow N, Day N. Statistical methods in cancer epidemiology, vol i: cohort studies. Lyon: International Agency for Research on Cancer; 1987.

13. United Nations Committee on Atomic Radiations (UNSCEAR). Report to the General Assembly. New York (NY): United Nations; 2000.

14. Gilbert ES, Fix JJ. Accounting for bias in dose estimates in analyses of data from nuclear worker mortality studies. Health Phys. 1995;68:650-60.

15. Xue X, Shore RE, Ye X, Kim MY. Estimating the dose response relationship for occupational radiation exposure measured with minimum detection level. Health Phys. 2004;87:397-404.

16. Rimpelä AH, Nurminen MM, Pulkkinen PO, Rimpelä MK, Valkonen T. Mortality of doctors: do doctors benefit from their medical knowledge? Lancet. 1987;1:84-6.

17. Pukkala E. Cancer risk by social class and occupation. Basel (Switzerland): Karger Press; 1995.

18. Juel K, Mosbech J, Hansen ES. Mortality and causes of death among Danish medical doctors 1973-1992. Int J Epidemiol. 1999;28:456-60.

19. Anderson NE, King SH, Miller KL. Variations in dose to the extremities of vascular/interventional radiologists. Health Phys. 1999;76 suppl 2:S39-40.

20. Saether HK. Davidson TM, Widmark A, Wohni T. Measurements of finger doses in $\mathrm{x}$-ray guided surgery, nuclear medicine and research. Radiat Prot Dosimetry. 2005;113:392-395.

Received for publication: 1 November 2005 